\begin{tabular}{|c|c|c|c|c|c|c|}
\hline & \multicolumn{2}{|c|}{$\mathrm{RA}(\mathrm{N}=201)$} & \multicolumn{2}{|c|}{$\operatorname{axSpA}(\mathrm{N}=134)$} & \multicolumn{2}{|c|}{ PsA (N=169) } \\
\hline & Mean (SD) & Q1, Q3 & Mean (SD) & Q1, Q3 & Mean (SD) & Q1, Q3 \\
\hline Age at SB5 initiation (years) & $60.2(11.7)$ & 53,68 & $50.5(13.6)$ & 38,61 & $53.0(12.2)$ & 43,62 \\
\hline \multirow[t]{2}{*}{ Duration of disease (years) } & $13.5(11.7)$ & $4.5,20$ & $18.7(13.2)$ & 9,25 & $12.7(9.9)$ & 4,20 \\
\hline & n & $\%$ & n & $\%$ & n & $\%$ \\
\hline Women & 144 & 71.6 & 41 & 30.6 & 77 & 45.6 \\
\hline SB5 Dosing regimen: & 149 & 74.1 & 120 & 89.6 & 156 & 92.3 \\
\hline \multicolumn{7}{|l|}{ Baseline 40mg Q2W } \\
\hline Week 48 40mg Q2W & 69 & 77.5 & 67 & 85.9 & 98 & 92.5 \\
\hline Baseline Other ${ }^{*}$ & 52 & 25.9 & 14 & 10.4 & 13 & 7.7 \\
\hline Week 48 Other* & 20 & 22.4 & 11 & 14.1 & 8 & 7.5 \\
\hline Disease Score & & & & & Tender Joint & Swollen Joint \\
\hline (paired patients) & DAS28 & FFbH & \multicolumn{2}{|c|}{ BASDAI } & Count & Count \\
\hline Baseline, $\mathrm{n}$, mean $(95 \% \mathrm{Cl})$ & $692.5(2.3-2.7) 22$ & $73.9(65.6-82.1)$ & 42 & $2.8(2.3-3.4)$ & $491.8(0.1-3.0)$ & $490.6(0.2-0.9)$ \\
\hline Week $48, \mathrm{n}$, mean $(95 \% \mathrm{Cl})$ & $692.6(2.3-2.8) 22$ & $72.1(64.0-80.2)$ & 42 & $3.0(2.4-3.7)$ & $491.9(0.5-3.3)$ & $490.6(0.1-1.1)$ \\
\hline \multicolumn{7}{|c|}{ Patient diagnosed with CoVID-19 at any time on-study, $n$ (\%) } \\
\hline No & 142 & 95.3 & 96 & 88.1 & 140 & 98.6 \\
\hline Yes & 3 & 2.0 & 1 & 0.9 & 1 & 0.7 \\
\hline Unknown & 4 & 2.7 & 12 & 11.0 & 1 & 0.7 \\
\hline \multicolumn{7}{|c|}{ Imraldi regimen stopped or changed due to COVID-19, regardless of diagnosis, $n(\%)$} \\
\hline No & 130 & 98.5 & 106 & 97.2 & 134 & 100 \\
\hline Yes & 2 & 1.5 & 3 & 2.8 & 0 & 0 \\
\hline
\end{tabular}

SD standard deviation; Q1 1st quartile, Q3 3rd quartile; CI Confidence Interval*Other includes all other reported doses and/or dosing intervals: 40mg QW, 80mg Q2W, and unspecified frequencyDAS28 Disease Activity Score 28; FFbH Hanover Functional Ability Questionnaire; BASDAI Bath Ankylosing Spondylitis Disease Activity Index

Background: SB5, a biosimilar to reference adalimumab (ADL), received EU marketing authorisation in August 2017, based on the totality of evidence from pre-clinical and clinical Phase I and III studies that demonstrated bioequivalence, similar efficacy, and comparable safety and immunogenicity to the reference. This real-world study provides data on outcomes of the transition from reference to biosimilar ADL outside the controlled, randomised, clinical trial setting. Objectives: To evaluate candidate predictors of persistence on SB5 in EU patients (pts) across multiple indications.

Methods: This ongoing observational study enrolled 1000 pts with rheumatoid arthritis (RA), axial spondyloarthritis (axSpA), psoriatic arthritis (PsA), ulcerative colitis, or Crohn's disease who initiated SB5 as part of routine clinical practice following a minimum of 16 weeks' treatment with reference ADL, at clinics in Belgium, Germany, Ireland, Italy, Spain, and the UK. Data are captured from clinic records retrospectively for the 24 weeks prior to transition, and prospectively and/or retrospectively for 48 weeks following transition. Primary outcome measures include baseline clinical characteristics, disease activity scores and clinical management over time; data on COVID-19 infection has recently been captured. This interim analysis (IA) provides an overview of baseline characteristics, disease scores and dose regimen up to 48 weeks post-initiation of SB5, and COVID-19 infection reported to date, in subjects with RA, axSpA, or PsA enrolled at 35 specialist sites and followed up to the data extract date of $18^{\text {th }}$ December 2020.

Results: Of the 504 pts included in this IA, 201 have RA, 169 have PsA, and 134 axSpA. At time of data extract, 216 pts had completed 48 weeks on SB5, 73 pts had discontinued SB5, and 8 had discontinued the study.

Conclusion: This IA provides a first insight into clinical management of pts over 48 weeks, in a contemporary cohort of EU pts with established RA, axSpA and PsA, switched from reference to biosimilar ADL SB5 in clinical practice. The majority of pts showed no meaningful difference in disease score or dose regimen of SB5 by Week 48 post-transition. As of data extract date, the proportion of pts with a known positive COVID-19 test was low (1.3\%) and a small minority $(1.3 \%)$ had SB5 treatment changed or interrupted as a result of the COVID-19 pandemic. With follow-up of pts ongoing to Q4 2021, the study will continue to provide pertinent information about clinical outcomes of transition from reference to biosimilar ADL in real-world practice and in indications not investigated in controlled studies.

Acknowledgements: Statistical services gave been provided by FGK Clinical Research $\mathrm{GmbH}$, Munich, Germany. Data management services were provided by Worldwide Clinical trial, Research Triangle Park, NC, USA; funding was provided by Biogen International $\mathrm{GmbH}$.

Disclosure of Interests: Ulf Müller-Ladner Consultant of: Biogen, Grant/ research support from: Biogen, Karl Gaffney Consultant of: AbbVie, Celgene, Gilead, Lilly, MSD, Novartis, Pfizer, and UCB, Grant/research support from: AbbVie, Celgene, Gilead, Lilly, MSD, Novartis, Pfizer, and UCB, Deepak Jadon Consultant of: AbbVie, Celgene, Eli Lilly, Gilead, Janssen, MSD, Novartis, Oxford University Press, Pfizer, Roche, Sandoz, UCB, Grant/research support from: AbbVie, Celgene, Eli Lilly, Gilead, Janssen, MSD, Novartis, Oxford University Press, Pfizer, Roche, Sandoz, UCB, Ulrich Freudensprung Shareholder of: Biogen, Employee of: Biogen, Janet Addison Shareholder of: Biogen, Employee of: Biogen

DOI: 10.1136/annrheumdis-2021-eular.844

\section{AB0205 \\ A NOVEL METHOD FOR PREDICTING 1-YEAR RETENTION OF ABATACEPT USING MACHINE LEARNING TECHNIQUES: DIRECTIONALITY AND IMPORTANCE OF PREDICTORS}

R. Alten ${ }^{1}$, C. Behar ${ }^{2}$, C. Boileau ${ }^{3}$, P. Merckaert ${ }^{4}$, E. Afari ${ }^{5}$, V. Vannier-Moreau ${ }^{6}$, S. Connolly ${ }^{7}$, A. Najm ${ }^{8}$, P. A. Juge ${ }^{9}$, A. Rai ${ }^{10}$, Y. Elbez ${ }^{11}$, K. Lozenski ${ }^{12}$.

${ }^{1}$ Schlosspark-Klinik University, Department of Internal Medicine, Rheumatology, Berlin, Germany; ${ }^{2} T u l s y$, Co-founder, Paris, France; ${ }^{3}$ Excelya, N/A, BoulogneBillancourt, France; ${ }^{4}$ Data Revenue $\mathrm{GmbH}$, Machine Learning Engineering Department, Berlin, Germany; ${ }^{5}$ Private Practice, N/A, Brunoy, France; ${ }^{6}$ Bristol Myers Squibb, Medical Affairs, France, Rueil-Malmaison, France; ${ }^{7}$ Bristol Myers Squibb, Global Drug Development, Princeton, United States of America; ${ }^{8}$ University of Glasgow, Institute of Infection, Immunity and Inflammation, College of Medical Veterinary and Life Sciences, Glasgow, United Kingdom; ${ }^{9}$ Université de Paris, AP-HP, Hôpital Bichat Claude-Bernard, Department of Rheumatology, Paris, France; ${ }^{10}$ Bristol Myers Squibb, Global Biometrics and Data Science, Princeton, United States of America; ${ }^{11}$ Deepscover, Biostatistics, Puteaux, France; ${ }^{12}$ Bristol Myers Squibb, Immunology and Fibrosis, Princeton, United States of America

Background: In the ACTION (NCT02109666) study, multivariable Cox proportional hazards regression models showed that the predictors of 1-year retention to abatacept treatment were: patient global pain assessment, country, reason for stopping last biologic, number of prior biologic treatments, abatacept monotherapy, RF/anti-cyclic citrullinated peptide (CCP) status, previous neoplasms, psychiatric disorders and cardiac disorders. ${ }^{1}$ Machine learning techniques, using the gradient-boosting model, subsequently identified additional predictors of abatacept retention in patients with moderate-to-severe RA enrolled in ACTION; however, the analysis did not show the directionality of the predictors. ${ }^{2}$

Objectives: To improve the clinical interpretability of the machine learning model in terms of directionality and the importance of each variable in predicting retention.

Methods: Previous analyses using the gradient-boosting model to identify predictors of abatacept retention at 1 year in the ACTION study have been described. ${ }^{2}$ This analysis used SHapley Additive exPlanations (SHAP), a mathematical framework, to show how a particular predictor value influences prediction in the context of all other predictors. Higher SHAP values indicate a higher likelihood of retention. The contribution of every variable in the model's prediction (with the exception of country variables) was computed for each data point to capture individual variable impact. This enabled interpretation for level of importance and directionality at a patient level.

Results: Using data from 2350 patients enrolled in ACTION (May 2008 to December 2013), the mean retention rate at 1 year was 59.3\% $(n=1393)$. Overall variable importance is shown in Figure 1. After removal of country variables, the top five baseline predictors of retention were: no previous corticosteroid use, ACR functional class $\mathrm{II}, \geq 2$ prior biologic treatments prior to abatacept initiation, abatacept monotherapy and HAQ-DI. In terms of directionality, no previous corticosteroid use, $\geq 2$ prior biologic treatments prior to abatacept initiation, abatacept monotherapy and a higher HAQ-DI score at baseline were associated with a lower likelihood of retention; ACR functional class II was associated with a higher likelihood of retention. 
Conclusion: The gradient-boosting model previously identified predictors of abatacept retention from ACTION; ${ }^{2}$ the addition of SHAP in this analysis has provided information on the importance and directionality of those predictors. The most important predictor of abatacept retention was no previous corticosteroid use, which was associated with lower retention. The models and predictors identified could be further refined by using additional datasets from clinical trials. Machine learning offers an innovative and complementary approach to biostatistics and could be used to identify treatment response predictors at an individual patient level, leading to a more personalised treatment approach.

\section{REFERENCES:}

[1] Alten R, et al. RMD Open 2017;3:e000538.

[2] Alten R, et al. Presented at the virtual ACR Convergence 2020; 5-9 November 2020. Poster number 1745

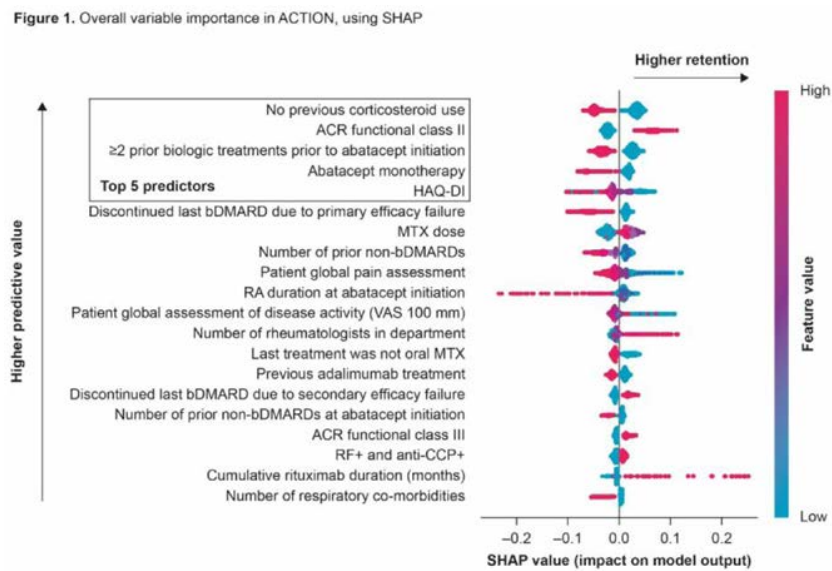

Colours indicate the value of the variable: pink represents higher and blue represents lower. The bulges in the plot indicate $\mathrm{mo}$ patients with that value; each dot represents a single patient. Higher SHAP values indicate a higher likelihood of retention.

Acknowledgements: This study was supported by Bristol Myers Squibb. Professional medical writing and editorial assistance was provided by Claire Line, PhD, at Caudex and was funded by Bristol Myers Squibb.

Disclosure of Interests: Rieke Alten Speakers bureau: AbbVie, Bristol Myers Squibb, Gilead, Janssen, Lilly, Pfizer, Consultant of: AbbVie, Bristol Myers Squibb, Gilead, Janssen, Lilly, Pfizer, Grant/research support from: AbbVie, Bristol Myers Squibb, Gilead, Janssen, Lilly, Pfizer, Claire Behar Shareholder of: I have not invested directly in pharmaceutical companies producing drugs/devices for use in rheumatology however I may have shares via the funds linked to my life insurance., Consultant of: Bristol Myers Squibb, Christine Boileau Consultant of: AstraZeneca, Bristol Myers Squibb, Nanobiotix, Pierre Merckaert Consultant of: Bristol Myers Squibb, Ebenezer Afari Consultant of: Bristol Myers Squibb, Virginie Vannier-Moreau Shareholder of: Bristol Myers Squibb, Employee of: Bristol Myers Squibb, Sean Connolly Shareholder of: Bristol Myers Squibb, Employee of: Bristol Myers Squibb, Aurelie Najm Speakers bureau: Bristol Myers Squibb, Consultant of: Bristol Myers Squibb, Pierre-Antoine Juge Consultant of: Bristol Myers Squibb, Angshu Rai Shareholder of: Amgen Inc, Consultant of: Amgen Inc, Employee of: Amgen Inc, Bristol Myers Squibb, Yedid Elbez Consultant of: Bristol Myers Squibb, Karissa Lozenski Shareholder of: Bristol Myers Squibb, Employee of: Bristol Myers Squibb

DOI: 10.1136/annrheumdis-2021-eular.868

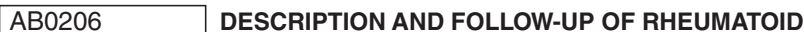 ARTHRITIS PATIENTS WHO STOPPED B/TSDMARD THERAPY, STRATIFIED BY CESSATION REASON}

T. Burkard $^{1}$, E. Vallejo-Yagüe ${ }^{1}$, T. Hügle ${ }^{2}$, A. Finckh ${ }^{3}$, A. M. Burden ${ }^{1,4} .{ }^{1} E T H$ Zurich, Department of Chemistry and Applied Biosciences, Zurich, Switzerland; ${ }^{2}$ Lausanne University Hospital, Department of Rheumatology, Lausanne, Switzerland; ${ }^{3}$ University Hospitals Geneva, Division of Rheumatology, Geneva, Switzerland; ${ }^{4}$ Maastricht University Medical Center+, Department of Clinical Pharmacy and Toxicology, Maastricht, Netherlands

Background: Biologic or targeted synthetic disease modifying antirheumatic drugs (b/tsDMARD) may be stopped for several reasons such as non-response, adverse events, remission, or other reasons (e.g. major surgery). Understanding the reasons and consequences of b/tsDMARD therapy cessation may contribute towards therapy decision guidance. Moreover, identifying patient characteristics leading to the re-start of b/tsDMARD therapy may guide decision-making as to which patients should remain on continuous b/tsDMARD therapy versus who may potentially stop b/tsDMARD therapy.
Objectives: To describe and follow rheumatoid arthritis (RA) patients who stopped b/tsDMARD therapy, stratified by cessation reason.

Methods: We conducted a descriptive cohort study among adult RA patients in the longitudinal Swiss Clinical Quality Management in Rheumatic Diseases (SCQM) between 1997 and 2019. RA patients who stopped their first b/tsDMARD therapy were eligible, with therapy stop date defining cohort entry. We followed all eligible patients from cohort entry until b/tsDMARD re-start (the outcome) or censoring due to end of patient record. All analyses were carried out stratified by cessation reason (non-response, adverse events, remission, othe reasons, unknown reasons). We described patient characteristics (demographics, lifestyle factors, clinical information, other medication use, relevant comorbidities) at cohort entry. Furthermore, we estimated Kaplan Meier curves to describe differences in cumulative incidences of b/tsDMARD re-start. Finally, we assessed patient characteristics at b/tsDMARD re-start and compared them with those at cohort entry.

Results: Among 2559 eligible RA patients, the majority stopped their b/tsDMARD due to non-response $(982,38 \%)$, followed by adverse events $(475,19 \%)$ other reasons $(445,17 \%)$, unknown reasons $(444,17 \%)$, and remission $(213$, $8 \%$ ). Mean age at b/tsDMARD stop was around 56.2 years except in patients who stopped due to remission (mean age of 58.1 years). The majority of patients were women $(78 \%)$, stopping due to an adverse event had the highest proportion of women (84\%), stopping due to remission had the lowest proportion of women (70\%). Compared to patients who stopped b/tsDMARD therapy due to non-response or adverse events, patients who stopped due to remission were generally more physically active, better educated, less likely to have a family history of rheumatic diseases, and had shorter median disease duration. A total of 2086 patients $(82 \%)$ re-started b/tsDMARD therapy during follow-up. Of these, the majority did so after stopping due to non-response (94\%), followed by adverse events $(82 \%)$, unknown reasons $(79 \%)$, other reasons $(74 \%)$, and remission $(47 \%)$. The median cumulative incidence of re-starting b/tsDMARD therapy was shortest after non-response (30 days), followed by unknown reasons (31 days), adverse events (94 days), other reasons (212 days), and remission (1597 days) The population who stopped b/tsDMARD therapy due to remission or other reasons yielded increased RA disease activity and an increase in proportions of women, cardiac diseases, degenerative joint disease, other auto-immune diseases, and of patients with family history of rheumatic diseases at the date of b/ tsDMARD re-start. However, among patients who stopped b/tsDMARD therapy due to non-response or adverse events, patient characteristics at b/tsDMARD re-start were unchanged compared to those at b/tsDMARD stop.

Conclusion: Observed differences in patient characteristics at b/tsDMARD stop may yield insight into why the patient was not responding, had an adverse event or achieved remission. Observed changes in patient characteristics from the date of $b / t s D M A R D$ stop to re-start identified which ones may lead to a worsening of RA activity in the absence of b/tsDMARD therapy.

Acknowledgements: We would like to thank Dr. Almut Scherer, Monika Hebeisen, and Eleftherios Papagiannoulis from SCQM for providing the data and answering questions thereto. A list of rheumatology offices and hospitals that are contributing to the SCQM registries can be found on www.scqm.ch/institutions. The SCQM is financially supported by pharmaceutical industries and donors. A list of financial supporters can be found on www.scqm.ch/sponsors.

Disclosure of Interests: Theresa Burkard: None declared, Enriqueta Vallejo-Yagüe: None declared, Thomas Hügle Consultant of: Pfizer, Abbvie, Novartis, Grant/research support from: GSK, Jansen, Pfizer, Abbvie, Novartis, Roche, MSD, Sanofi, BMS, Eli Lilly, UCB, Axel Finckh Speakers bureau: Pfizer, Eli-Lilly Paid instructor for: Pfizer, Eli-Lilly, Consultant of: Pfizer, BMS, Novartis, Grant/ research support from: AbbVie, AB2Bio, BMS, Gilead, Pfizer, Viatris, Andrea Michelle Burden: None declared DOI: 10.1136/annrheumdis-2021-eular.898

\section{$\mathrm{AB} 0207$ \\ ANALYSIS OF ABATACEPT TREATMENT RETENTION AND EFFICACY ACCORDING TO DISEASE DURATION AND TREATMENT LINE IN A REAL-WORLD SETTING}

R. Alten ${ }^{1}$, X. Mariette ${ }^{2}$, R. M. Flipo ${ }^{3}$, R. Caporali ${ }^{4,5}$, M. H. Buch ${ }^{6,7}$,

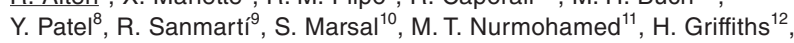
P. Peichl ${ }^{13}$, B. Bannert ${ }^{14}$, A. Forster ${ }^{15}$, M. Chartier ${ }^{16}$, Y. Elbez $^{17}$, C. Rauch ${ }^{18}$, V. Khaychuk ${ }^{19}$, K. Lozenski ${ }^{20} .{ }^{1}$ Schlosspark-Klinik University, Department of Internal Medicine, Rheumatology, Berlin, Germany; ${ }^{2}$ Université ParisSaclay, AP-HP, Hospital Bicêtre, Department of Rheumatology, Paris, France; ${ }^{3}$ Centre Hospitalier Universitaire de France, Department of Rheumatology, Lille, France; ${ }^{4}$ University of Milan, Department of Clinical Sciences and Community Health, Milan, Italy; ${ }^{5}$ G. Pini Hospital, Clinical Rheumatology Unit, Milan, Italy; ${ }^{6}$ University of Leeds, Leeds Institute for Rheumatic and Musculoskeletal Medicine, Leeds, United Kingdom; ${ }^{7}$ University of Manchester, Division of Musculoskeletal \& Dermatological Sciences, Manchester, United Kingdom; ${ }^{8}$ Hull Royal Infirmary, Department of Rheumatology, Hull, United Kingdom; ${ }^{9}$ Hospital Clinic de Barcelona, 